\title{
Kinds and Distribution of Pythium Species Isolated from Soils in Tohoku District*
}

\author{
Tsuneo WATANABE**
}

Key words: Pythium spp., kinds, distribution, Tohoku district.

Pythium species were obtained from soils and plant roots in various habitats in Tohoku district and the Ryukyu Islands in Japan with a special emphasis on isolation procedures, and also studied for the populations in soil quantitatively in the previous repor $\mathrm{t}^{1)}$. This is a further study on the kinds and distribution of Pythium species in Tohoku district in relation to the previous reports ${ }^{1-5}$. A total of 28 soil samples (Table 1) were collected in September, 1978 and August, 1981. Soil sampling and isolation method were described previously1). The fungi were cultured on various media including Difco corn meal agar for mycological study. Several soaking solutions including Petri's salt solution were used to study asexual reproduction. All of the fungi were identified following the descriptions by the previous workers ${ }^{6,7)}$.

A total of 210 Pythium isolates were obtained from soils from 26 locations in Tohoku district, and were identified into 14 taxa including H-Zs (a provisional Pyhtium group with zoospore formation from hypha-like sporangia, but without sexual organ) and 13 unidentified isolates (Table 2). No Pythium was isolated from two samples (Sample 8 and 24) collected from forest soil near Lake Towada at Kosaka, Akita Pref. and musk melon field at Tendo, Yamagata, but the respective of 26 samples yielded one to five taxa. Most of these taxa were recorded in soils of different parts of Japan ${ }^{4,5,8}$ and other countries ${ }^{9-13)}$. Pythium taxa with numbers of samples yielding each taxon, and of their isolates obtained are listed, and representative isolates and their origin are summarized in Table 2.

Four Pythium taxa, i.e., $P$. dissotocum Drechsler, $P$. hydnosporum (Mont.) Schroeter, $P$. inflatum Matthews and H-Zs were identified in this study, and included in the previous Pythium list in Tohoku district ${ }^{1}$.

Of the 13 unidentified isolates, Isolate $78-2310$ and four others were sterile, forming just aseptate Pythium-like hyphae, and seven including Isolate 78-2241 formed lobate hyphal swellings without zoospore discharge. Another isolate (Isolate 78-2237) is close to P. carolinianum Matthews forming sphaerosporangia without internal proliferation, although zoospore discharge has been very rarely observed.

* Part of this work was conducted at National Institute of Agricultural Sciences (presently National Institute of Agro-Environmental Sciences).

** Forestry and Forest Products Research Institute, P.O. Box 16, Tsukuba Science City, Ibaraki 305, Japan 農林水産省森林総合研究所

1) Watanabe, T. (1981). Ann. Phytopath. Soc. Japan 47: 449-456. 2) Watanabe, T. (1981). Ibid. 47: 562-565. 3) Watanabe, T. (1983). Trans. mycol. Soc. Japan 24: 15-23. 4) Watanabe, T. (1988). Ann. Phytopath. Soc. Japan 54: 523-528. 5) Watanabe, T. (1989). Ibid. 55: 32-40. 6) Middleton, J.T. (1943). Mem. Torrey Botanical Club 20: 1-171. 7) Plaats-Niterink, A.J. van der (1981). Monograph of the genus Pythium. Studies in Mycology (CBS) 21: 1-242. 8) Watanabe, T. (1985). Plant Prot. Bull. (Taiwan, R.O.C.) 27: 211-224. 9) Dick, M.W. and Ali-Shtayeh, M.S. (1986). Trans. Br. mycol. Soc. 86: 49-62. 10) Hendrix, F.F., Jr. and Campbell, W.A. (1970). Can. J. Bot. 48: $377-384$. 11) Vaartaja, O. and Bumbieris, M. (1964). Aust. J. Biol. Sci. 17: 436-445. 12) Campbell, W.A. and Hendrix, F.F., Jr. (1967). Mycologia 59: 274-278. 13) Hendrix, F.F., Jr. and Campbell, W.A. (1968). Forest Science 14: 292-297. 
Table 1. Soil samples obtained from 28 different locations with various habitats and soil $\mathrm{pH}$ values in Tohoku district, Japan

\begin{tabular}{|c|c|c|c|c|c|c|c|}
\hline Sample & Location & Habitat & $\mathrm{pH}$ & Sample & Location & Habitat & $\mathrm{pH}$ \\
\hline \multicolumn{4}{|c|}{ Aomori Pref. } & \multicolumn{4}{|c|}{ Akita Pref. } \\
\hline 9 & Towada & Forest & 4.6 & 1 & Akita & Strawberry & 4.7 \\
\hline 10 & Towada & Forest & 4.6 & 2 & Taiyuu & Cucumber & 4. 6 \\
\hline 11 & Kuroishi & Chinese cabbage & 4.6 & 3 & Taiyuu & Japanese hornwort & 4.7 \\
\hline 12 & Kuroishi & Buckwheat & 4.6 & 4 & Taiyuu & Japanese hornwort & 4.8 \\
\hline 13 & Iwaki & Fallow (corn)a) & 4.7 & 53 & Taiyuu & Japanese hornwort & 4.7 \\
\hline 14 & Ajigasawa & Fallow (watermelon) & 4.6 & 6 & Omonogawa & Fallow (watermelon) & 4.6 \\
\hline 15 & Kizukuri & Fallow (watermelon) & 4.8 & 7 & Omonogawa & Fallow (watermelon) & 4.6 \\
\hline 16 & Kizukuri & Peanut & 4.6 & 8 & Kosaka & Forest & 5.0 \\
\hline 17 & Kizukuri & Peanut & 4.6 & & & & \\
\hline \multirow[t]{2}{*}{18} & Hiraka & Radish & 4.8 & \multicolumn{4}{|c|}{ Yamagata Pref. } \\
\hline & & & & 24 & Tendou & Musk melon & 4.7 \\
\hline \multicolumn{4}{|c|}{ Iwate Pref. } & $25 \mathrm{~s}$ & Yamagata & Cucumber & 4.4 \\
\hline 19 & Morioka & Tomato & 4.7 & 26 & Yamagata & Welsh onion & 4.6 \\
\hline 20 & Morioka & Strawberry & 4.6 & $27 \mathrm{~s}$ & Yamagata & Buckwheat & 4.7 \\
\hline 21 & Morioka & Apple & 4.7 & & & & \\
\hline $22 s$ & Morioka & Alfalfa & 4.5 & \multicolumn{4}{|c|}{ Fukushima Pref. } \\
\hline 23 & Tarou & Fallow & 4.6 & 94 & Kitashiobara & Eggplant & 5.8 \\
\hline
\end{tabular}

a) ( ): previous crop.

Table 2. List of Pythium taxa isolated from soils of Tohoku district and numbers of soil samples yielding each taxon and of their total isolates in each taxon with representative isolates and their origin

\begin{tabular}{|c|c|c|c|}
\hline Species & $\begin{array}{c}\text { No. of } \\
\text { samples }\end{array}$ & $\begin{array}{l}\text { No. of } \\
\text { isolates }\end{array}$ & $\begin{array}{l}\text { Representative isolates } \\
\text { and their origin }\end{array}$ \\
\hline P. aphanidermatum (Edson) Fitz. & 6 & 12 & 78-2255 (Sample 20) \\
\hline P. dissotocum Drechsler & 2 & 3 & 78-2428 (Sample 4) \\
\hline P. hydnosporum (Mont.) Schroeter & 1 & 2 & 81-256 (Sample 94) \\
\hline P. inflatum Matthews & 2 & 2 & 78-2324 (Sample 13) \\
\hline$P$. intermedium de Bary & 2 & 3 & 78-2313 (Sample 10) \\
\hline P. irregulare Buisman & 4 & 8 & 78-2453 (Sample 17) \\
\hline$P$. periplocum Drechsler & 1 & 1 & 78-2243 (Sample 15) \\
\hline$P$. spinosum Sawada & 15 & 37 & 78-2357 (Sample 22) \\
\hline P. splendens Braun & 3 & 3 & 78-2259 (Sample 26) \\
\hline$P$. sylvaticum Campbell et Hendrix & 23 & 94 & 78-2222 (Sample 1) \\
\hline P. torulosum Coker et Patterson & 3 & 4 & 78-2307 (Sample 7) \\
\hline P. ultimum Trow & 6 & 17 & 78-2314 (Sample 11) \\
\hline P. vexans de Bary & 1 & 1 & 78-2360 (Sample 25) \\
\hline $\mathrm{H}-\mathrm{Zs}$ a) & 3 & 10 & 78-2244 (Sample 15) \\
\hline Pythium spp. & 9 & 13 & 78-2323 (Sample 13) \\
\hline
\end{tabular}

a) A provisional Pythium group with zoospore formation from hypha-like sporangia, but without sexual organs. 
Table 3. Kinds of Pythium species and number of isolates obtained from soils of 28 different locations in Tohoku district

\begin{tabular}{|c|c|c|c|c|c|c|c|c|c|c|c|c|}
\hline \multirow{2}{*}{$\begin{array}{l}\text { Sample } \\
\text { No. }\end{array}$} & \multicolumn{12}{|c|}{ Kind of Pythium a) and no. of isolates } \\
\hline & aph & dis & $\inf$ & int & irr & spi & spl & syl & tor & ult & $\mathrm{H}-\mathrm{Zs}$ & Miscellaneous b) \\
\hline \multicolumn{13}{|c|}{ Aomori Pref. } \\
\hline 9 & & & & 2 & & & & 1 & & & & \\
\hline 10 & & & & 1 & & & & 3 & & & & \\
\hline 11 & 1 & & & & & & & 4 & & 3 & & Py. sp., 1 \\
\hline 12 & & & & & & 1 & & 4 & & & 3 & \\
\hline 13 & & & 1 & & & 4 & & 5 & 2 & & & Py. sp., 2 \\
\hline 14 & & & & & & 6 & & 1 & & 1 & & Py. sp., 1 \\
\hline 15 & & & & & & 1 & & 2 & & & 6 & per, 1 ; Py. sp., 4 \\
\hline 16 & & & & & 1 & & & 2 & & & & \\
\hline 17 & & & & & 3 & 2 & & & & & & \\
\hline 18 & & & & & & & & 4 & & & 1 & \\
\hline \multicolumn{13}{|l|}{ Iwate Pref. } \\
\hline 19 & 2 & & & & & & & 3 & & & & . \\
\hline 20 & 3 & & & & & 2 & & 7 & & & & \\
\hline 21 & & & & & & 1 & 1 & & & & & \\
\hline $22 ;$ & & & 1 & & & 3 & & 4 & & & & Py. sp., 1 \\
\hline 23 & & & & & 3 & & & 2 & & & & \\
\hline \multicolumn{13}{|c|}{ Akita Pref. } \\
\hline 1. & 1 & & & & & 2 & & 7 & & 2 & & \\
\hline 2 & & & & & & 6 & & 6 & & & & \\
\hline 3 & & 2 & & & & 1 & & 6 & & & & \\
\hline 4 & & 1 & & & & & & 1 & & & & \\
\hline $5 i$ & & & & & & 1 & & 8 & & & & Py. sp., 1 \\
\hline 6 & & & & & & 5 & & 1 & & & & Py. sp., 1 \\
\hline 7 & & & & & & 1 & 1 & 4 & 1 & & & Py. sp., 1 \\
\hline 8 & & & & & & & & & & & & \\
\hline \multicolumn{13}{|c|}{$\begin{array}{l}\text { Yamagata Pref. } \\
24\end{array}$} \\
\hline $25 \mathrm{~s}$ & 3 & & & & & & & 10 & 1 & 7 & & vex, 1 \\
\hline 26 & & & & & & & 1 & 3 & & 1 & & \\
\hline $27 \mathrm{~s}$ & 2 & & & & & 1 & & 6 & & 3 & & Py. sp., 1 \\
\hline Fukushime & a Pref & & & & & & & & & & & \\
\hline 94 & & & & & 1 & & & & & & & hyd, 2 \\
\hline Total isol. & 12 & 3 & 2 & 3 & 8 & 37 & 3 & 94 & 4 & 17 & 10 & 17 \\
\hline
\end{tabular}

a) Abbreviated as follows: aph, $P$. aphanidermatum; dis, $P$. dissotocum; hyd, $P$. hydnosporum; inf, $P$. inflatum; int, P. intermedium; irr, P. irregulare; per, P. periplocum; spi, P. spinosum; spl, P. splendens; syl, $P$. sylvaticum; tor, $P$. torulosum; ult, $P$. ultimum; vex, $P$. vexans; $\mathrm{H}-\mathrm{Zs}$, a provisional $P$ ythium group with zoospore formation from hypha-like sporangia, but without sexual organs.

b) Included Pythium taxa obtained only from one location (sample), and Py. sp. (=unidentified Pythium species).

Pythium sylvaticum was predominant, and isolated from 23 locations. More than $87 \%$ (82 isolates) of the 94 isolates obtained were heterothallic ${ }^{12,13}$.

Abundant occurrence of $P$. spinosum (Tables 2 and 3 ) is noteworthy, and isolated from 15 locations in Tohoku district. In addition, 12 isolates of $P$. aphanidermatum, high-temperature favorite Pythium, were obtained from six locations, including one isolate each from strawberry field in Akita city (Isolate 78-2223) and Chinese cabbage field in Kuroishi, Aomori Pref. (Isolate 78-2269).

I wish to thank Mr. Jiro Kobayashi, Akita Prefectural Agricultural Experiment Station, Mr. Zyunitsu 
Chiba, Aomori Prefectural Agricultural Experiment Station, Mr. Seiichi Hirayama, Yamagata Prefectural Agricultural Experiment Station and Mr. Manabu Umekawa, National Vegetable Experiment Station, Morioka Branch for help in collecting soil samples.

\section{和 文 摘 要}

渡辺恒雄 : 東北地方の土㙥から分離したPythium 菌の種と分布

東北地方の土㙥に生息するPythium 菌を，キュウリ種子などによる捕捉法と直接接種法を用いて分離し， 種と分布を調查した。供試した 28 カ所の土壤 ( 1 10 試料/県) のうち, 十和田湖周辺 (秋田県小坂町) の 林地と山形県天童市のマスクメロン畑からの 2 試料（試料 8 と 24) を除く各試料からは 1 ～種のPythium 菌が分離でき，合計 210 菌株を得たが，これらは，H-Zs（糸状胞子囊から遊走子を形成するが，生殖器官 は未形成の一群）を含む 14 種に分類・同定できた。最も多く分離されたのは日本の他の地方と同様に $P$. sylvaticum で，とくに雌雄異株が全分離株の $87 \%$ 以上を占めた。また P. spinosum の広い分布と好高温性 菌の P. aphanidermatum が秋田, 青森の両県を含む東北地方から高い頻度で分離されたことは注目に值す る。

(Received August 14, 1989) 